\title{
The curvature of contact structures on 3-manifolds
}

\author{
VLADIMIR KROUGLOV
}

\begin{abstract}
We study the sectional curvature of plane distributions on 3-manifolds. We show that if a distribution is a contact structure it is easy to manipulate its curvature. As a corollary we obtain that for every transversally oriented contact structure on a closed 3-dimensional manifold, there is a metric such that the sectional curvature of the contact distribution is equal to -1 . We also introduce the notion of Gaussian curvature of the plane distribution. For this notion of curvature we get similar results.
\end{abstract}

$53 \mathrm{D} 35 ; 53 \mathrm{~B} 21$

\section{Introduction}

The problem of prescribing the curvatures of a manifold is one of the central problems in Riemannian geometry. That is, given a smooth function can it be realized as a scalar (Ricci or sectional) curvature of some Riemannian metric on a manifold. The solution of the Yamabe problem is the best known result in prescribing the scalar curvature on a manifold (cf Lee and Parker [4]). There are several results on prescribing the Ricci curvature of a manifold (cf for example Lohkamp [5]). It is natural to ask to what extent it is possible to prescribe the sectional curvature of the plane distribution on a 3-manifold. It turns out that this problem is closely connected with the contactness of the distribution. In fact we have the following:

Theorem A Let $\xi$ be a transversally orientable contact structure on a closed orientable 3-manifold $M$. For any smooth strictly negative function $f$, there is a metric on $M$ such that $f$ is the sectional curvature of $\xi$.

If we impose more topological restrictions on the distribution we can obtain an even stronger result:

Theorem B Let $\xi$ be a transversally orientable contact structure on $M$ with Euler class zero. Then for any smooth function $f$, there is a metric on $M$ such that $f$ is a sectional curvature of $\xi$. 
In [2], Chern and Hamilton studied a similar problem of prescribing the so-called Webster curvature $W$ on a contact three-manifold. The main difference in their approach is that they restrict the class of metrics to the metrics which are adapted to a contact structure, while we deal with the class of all metrics. They prove that in their class one can either find a metric with the constant negative Webster curvature or a metric with strictly positive Webster curvature.

It is a well-known problem whether a foliation on a 3-dimensional manifold admits a simultaneous uniformization of all its leaves. The Reeb stability theorem asserts that on a compact orientable 3-manifold the only foliation with the leaves having positive Gaussian curvature is the foliation of $M=S^{2} \times S^{1}$ by spheres. It is known (see Candel [1]) that if $M$ is atoroidal and aspherical and the foliation is taut, then there is a metric on $M$ such that all leaves have constant negative Gaussian curvature -1 . In the case of contact structures we ask a similar question. For this we have to introduce the notion of Gaussian curvature of the plane distribution.

We define the Gaussian curvature of the plane distribution as the sum $K_{G}(\xi)=$ $K(\xi)+K_{e}(\xi)$ of the sectional and the extrinsic curvatures of the distribution. In the case of integrable $\xi$ this equation is nothing but the Gauss equation.

Definition 1.1 Let $\xi$ be a plane distribution on $M$. We say that $\xi$ admits a uniformization if there is a metric on $M$ such that the Gaussian curvature of $\xi$ is constant.

It turns out that unlike the case of foliations, every transversally orientable contact structure on a closed 3-manifold admits a uniformization. We have the following:

Theorem C Let $\xi$ be a transversally orientable contact structure on a closed orientable 3-manifold $M$. For any smooth strictly negative function $f$, there is a metric on $M$ such that $f$ is the Gaussian curvature of $\xi$.

This paper is organized as follows. In Section 2 we recall basic facts about the geometry of plane distributions. In Section 3 we prove the main technical lemma. Section 4 is devoted to the proof of Theorem A and Theorem B. We prove Theorem C in Section 5.

Acknowledgment I would like to thank Patrick Massot for pointing out Corollary 3.6. This led to a much stronger and natural formulation of Theorem B.

\section{Basic definitions and notation}

Throughout this paper $M$ will be a closed orientable 3-manifold. A distribution on $M$ is a two dimensional subbundle of the tangent bundle of $M$. That is, at each point $p$ 
in $M$ there is a plane $\xi_{p}$ in the tangent space $T_{p} M$. A distribution is called integrable, if there is a foliation on $M$ which is tangent to it. The following Frobenius theorem gives necessary and sufficient conditions for $\xi$ to be integrable.

Theorem 2.1 Let $\xi$ be a distribution on $M$. Then $\xi$ is integrable if and only if for any two sections $S$ and $T$ of $\xi$ its Lie bracket belongs to $\xi$.

Definition 2.2 A distribution $\xi$ is called a contact structure if for any linearly independent sections $S$ and $T$ of $\xi$ and for any $p \in M$ the Lie bracket $[S, T]$ at $p$ does not belong to $\xi_{p}$.

A distribution $\xi$ is called transversally oriented if there is a globally defined 1-form $\alpha$ such that $\xi=\operatorname{Ker}(\alpha)$. This is equivalent to say that there exists a globally defined vector field $n$ which is transverse to $\xi$. It is an easy consequence of Frobenius Theorem that $\xi$ is a contact structure if and only if

$$
\alpha \wedge d \alpha \neq 0
$$

Fix some orientation on $M$. A contact structure is said to be positive (resp. negative) if the orientation induced by $\alpha \wedge d \alpha$ coincides (resp. is opposite to) the orientation on $M$. A contact structure $\xi$ is called overtwisted, if there is an embedded disk such that $\left.T D\right|_{\partial D}=\left.\xi\right|_{\partial D}$. If $\xi$ is not overtwisted, it is called tight.

The Euler class $e(\xi) \in H^{2}(M, \mathbb{Z})$ of a plane distribution is the Euler class of the bundle $\xi \rightarrow M$. It is known that if $\xi$ is a 2 -dimensional plane distribution on $M$ with vanishing Euler class then $\xi$ is trivial. Recall, that a framing of $M$ is the presentation of the tangent bundle of $M$ as a product $T M \simeq M \times \mathbb{R}^{3}$. A framing on $M$ consists of three linearly independent vector fields. It is known that every closed orientable 3-manifold admits a framing.

A bi-contact structure on $M$ is a pair $(\xi, \eta)$ of transverse contact structures which define opposite orientation on $M$.

Assume that $M$ is a Riemannian manifold with the metric $\langle\cdot, \cdot\rangle$ and the Levi-Civita connection $\nabla$. Let $n$ be a local unit vector field orthogonal to $\xi$. We are now going to define the second fundamental form of $\xi$. The definition is due to Reinhart [7].

Definition 2.3 The second fundamental form of $\xi$ is a symmetric bilinear form, which is defined in the following way:

$$
B(S, T)=\frac{1}{2}\left\langle\nabla_{S} T+\nabla_{T} S, n\right\rangle
$$

for all sections $S$ and $T$ of $\xi$. 
Remark 2.4 If $\xi$ is integrable, then $B$ restricted to the leaf of $\xi$ agrees with the second fundamental form of the leaf.

Let $S$ and $T$ be two linearly independent sections of $\xi$.

Definition 2.5 We call the function

$$
K_{e}(\xi)=\frac{B(S, S) B(T, T)-B(S, T)^{2}}{\langle S, S\rangle\langle T, T\rangle-\langle S, T\rangle^{2}}
$$

an extrinsic curvature of $\xi$.

It is easy to verify that $K_{e}(\xi)$ depends only on $\xi$, not on the actual choice of $S, T$ and $n$.

Definition 2.6 Consider the function $K(\xi)$ which assigns to a point $p \in M$ the sectional curvature of the plane $\xi_{p}$. We call this function the sectional curvature of $\xi$.

Definition 2.7 We call the sum $K_{G}(\xi)=K(\xi)+K_{e}(\xi)$ the Gaussian curvature of $\xi$.

Let $S, T$ and $U$ be the local sections of $T M$. Recall the Koszul formula for the Levi-Civita connection of $\langle\cdot, \cdot\rangle$ :

$$
\begin{aligned}
2\left\langle\nabla_{S} T, U\right\rangle=S\langle T, U\rangle+T\langle U, S\rangle & -U\langle S, T\rangle \\
& +\langle[S, T], U\rangle-\langle[S, U], T\rangle-\langle[T, U], S\rangle
\end{aligned}
$$

\section{The deformation of metric}

In this section we will give the proof of the main technical results we will need throughout the paper.

Let $\xi$ be a transversally orientable plane distribution on a 3-dimensional Riemannian manifold $(M,\langle\cdot, \cdot\rangle)$. Fix a unit normal vector field $n$. Suppose $a$ is a strictly positive smooth function on $M$. A stretching of $\langle\cdot, \cdot\rangle$ along $n$ by the function $a$ is the following Riemannian metric on $M$ :

$$
\langle\cdot, \cdot\rangle_{a}=\left.\left.a\langle\cdot, \cdot\rangle\right|_{n} \oplus\langle\cdot, \cdot\rangle\right|_{\xi}
$$

Our aim is to calculate the sectional curvature of $\xi$ in the stretched metric in terms of the initial metric. 
Consider an open subset $U \subset M$ such that $\left.\xi\right|_{U}$ is a trivial fibration. Let $X$ and $Y$ be a pair of orthonormal sections of $\left.\xi\right|_{U}$. The triple $(X, Y, n)$ is an orthonormal framing on $U$ with respect to $\langle\cdot, \cdot\rangle$.

In the stretched metric this frame is orthogonal, vector fields $X$ and $Y$ are unit and the length of $n$ is equal to $\sqrt{a}$. Denote by $\nabla$ the Levi-Civita connection of $\langle\cdot, \cdot\rangle_{a}$.

Lemma 3.1 The sectional curvature of $\xi$ with respect to $\langle\cdot, \cdot\rangle_{a}$ can be calculated by the following formula:

$$
K(\xi)=-\frac{3}{4} a\langle[X, Y], n\rangle^{2}+P+\frac{1}{a} Q
$$

where

$$
\begin{aligned}
P= & X\langle[X, Y], Y\rangle-Y\langle[X, Y], X\rangle-\langle[X, Y], X\rangle^{2}-\langle[X, Y], Y\rangle^{2} \\
& \left.+\frac{1}{2}\langle[X, Y], n\rangle(-\langle[n, Y], X\rangle+\langle[n, X], Y\rangle)\right)
\end{aligned}
$$

and

$$
Q=\frac{1}{4}(\langle[X, n], Y\rangle+\langle[Y, n], X\rangle)^{2}-\langle[Y, n], Y\rangle\langle[X, n], X\rangle
$$

Proof Since $X$ and $Y$ are unit, the sectional curvature of $\xi$ is calculated by the formula:

$$
K(\xi)=\langle R(X, Y) Y, X\rangle_{a}=\left\langle\nabla_{X} \nabla_{Y} Y, X\right\rangle_{a}-\left\langle\nabla_{Y} \nabla_{X} Y, X\right\rangle_{a}-\left\langle\nabla_{[X, Y]} Y, X\right\rangle_{a}
$$

The first summand can be rewritten:

$$
\left\langle\nabla_{X} \nabla_{Y} Y, X\right\rangle_{a}=X\left\langle\nabla_{Y} Y, X\right\rangle_{a}-\left\langle\nabla_{Y} Y, \nabla_{X} X\right\rangle_{a}
$$

Apply the Koszul formula to $X\left\langle\nabla_{Y} Y, X\right\rangle_{a}$. We get:

$$
\begin{aligned}
X\left\langle\nabla_{Y} Y, X\right\rangle_{a} & =\frac{1}{2} X\left(2 Y\langle Y, X\rangle_{a}-X\langle Y, Y\rangle_{a}+\langle[Y, Y], X\rangle_{a}-2\langle[Y, X], Y\rangle_{a}\right) \\
& =-X\langle[Y, X], Y\rangle_{a}=-X\langle[Y, X], Y\rangle
\end{aligned}
$$

Decompose the vector field $\nabla_{Y} Y$ with respect to the frame $(X, Y, n / \sqrt{a})$ orthonormal in the stretched metric $\langle\cdot, \cdot\rangle_{a}$ :

$$
\nabla_{Y} Y=\left\langle\nabla_{Y} Y, \frac{n}{\sqrt{a}}\right\rangle_{a} \frac{n}{\sqrt{a}}+\left\langle\nabla_{Y} Y, Y\right\rangle_{a} Y+\left\langle\nabla_{Y} Y, X\right\rangle_{a} X
$$

Substituting these expressions into $\left\langle\nabla_{X} \nabla_{Y} Y, X\right\rangle_{a}$, we obtain:

$$
\begin{aligned}
\left\langle\nabla_{X} \nabla_{Y} Y, X\right\rangle_{a}=-X\langle[Y, X], Y\rangle-\left\langle\left\langle\nabla_{Y} Y, n\right\rangle_{a} \frac{n}{a}\right. & +\left\langle\nabla_{Y} Y, Y\right\rangle_{a} Y \\
& \left.+\left\langle\nabla_{Y} Y, X\right\rangle_{a} X, \nabla_{X} X\right\rangle_{a}
\end{aligned}
$$


Since $X$ and $Y$ are of unit length this reduces to:

$$
\left\langle\nabla_{X} \nabla_{Y} Y, X\right\rangle_{a}=-X\langle[Y, X], Y\rangle-\frac{1}{a}\left\langle\nabla_{Y} Y, n\right\rangle_{a}\left\langle\nabla_{X} X, n\right\rangle_{a}
$$

Apply the Koszul formula to the term $\left\langle\nabla_{Y} Y, n\right\rangle_{a}\left\langle\nabla_{X} X, n\right\rangle_{a}$. Finally, we have:

$$
\begin{aligned}
\left\langle\nabla_{X} \nabla_{Y} Y, X\right\rangle_{a} & =-X\langle[Y, X], Y\rangle-\frac{1}{a}\langle[Y, n], Y\rangle_{a}\langle[X, n], X\rangle_{a} \\
& =-X\langle[Y, X], Y\rangle-\frac{1}{a}\langle[Y, n], Y\rangle\langle[X, n], X\rangle
\end{aligned}
$$

The second summand is equal to:

$$
\begin{aligned}
-\left\langle\nabla_{Y} \nabla_{X} Y, X\right\rangle_{a}= & -Y\left\langle\nabla_{X} Y, X\right\rangle_{a}+\left\langle\nabla_{X} Y, \nabla_{Y} X\right\rangle_{a} \\
= & Y\left\langle Y, \nabla_{X} X\right\rangle_{a}+\left\langle\left\langle\nabla_{X} Y, n\right\rangle_{a} \frac{n}{a}+\left\langle\nabla_{X} Y, Y\right\rangle_{a} Y\right. \\
& \left.+\left\langle\nabla_{X} Y, X\right\rangle_{a} X, \nabla_{Y} X\right\rangle_{a} \\
= & -Y\langle[X, Y], X\rangle_{a}+\frac{1}{a}\left\langle\nabla_{X} Y, n\right\rangle_{a}\left\langle\nabla_{Y} X, n\right\rangle_{a}
\end{aligned}
$$

Write the equations for the terms $\left\langle\nabla_{X} Y, n\right\rangle_{a}$ and $\left\langle\nabla_{Y} X, n\right\rangle_{a}$ :

$$
\begin{aligned}
2\left\langle\nabla_{X} Y, n\right\rangle_{a} & =\langle[X, Y], n\rangle_{a}-\langle[X, n], Y\rangle_{a}-\langle[Y, n], X\rangle_{a} \\
& =a\langle[X, Y], n\rangle-\langle[X, n], Y\rangle-\langle[Y, n], X\rangle \\
2\left\langle\nabla_{Y} X, n\right\rangle_{a} & =\langle[Y, X], n\rangle_{a}-\langle[Y, n], X\rangle_{a}-\langle[X, n], Y\rangle_{a} \\
& =a\langle[Y, X], n\rangle-\langle[Y, n], X\rangle-\langle[X, n], Y\rangle
\end{aligned}
$$

Inserting the above equations into the second summand we have:

$$
\begin{array}{r}
-\left\langle\nabla_{Y} \nabla_{X} Y, X\right\rangle_{a}=-Y\langle[X, Y], X\rangle_{a}+\frac{1}{4 a}(-a\langle[X, Y], n\rangle+\langle[X, n], Y\rangle+\langle[Y, n], X\rangle) \\
\cdot(-a\langle[Y, X], n\rangle+\langle[Y, n], X\rangle+\langle[X, n], Y\rangle)
\end{array}
$$

The last summand is:

$$
\begin{aligned}
-\left\langle\nabla_{[X, Y]} Y, X\right\rangle_{a}= & -\left\langle\nabla_{\langle[X, Y], n\rangle n+\langle[X, Y], X\rangle X+\langle[X, Y], Y\rangle Y} Y, X\right\rangle_{a} \\
= & -\langle[X, Y], n\rangle\left\langle\nabla_{n} Y, X\right\rangle_{a}-\langle[X, Y], X\rangle\left\langle\nabla_{X} Y, X\right\rangle_{a} \\
& -\langle[X, Y], Y\rangle\left\langle\nabla_{Y} Y, X\right\rangle_{a}
\end{aligned}
$$


The term $\left\langle\nabla_{n} Y, X\right\rangle_{a}$ is equal to

$$
\begin{aligned}
\left\langle\nabla_{n} Y, X\right\rangle_{a} & =-\frac{1}{2}\left(-\langle[n, Y], X\rangle_{a}+\langle[n, X], Y\rangle_{a}+\langle[Y, X], n\rangle_{a}\right) \\
& =-\frac{1}{2}(-\langle[n, Y], X\rangle+\langle[n, X], Y\rangle+a\langle[Y, X], n\rangle)
\end{aligned}
$$

which gives us:

$$
\begin{gathered}
-\left\langle\nabla_{[X, Y]} Y, X\right\rangle_{a}=-\langle[X, Y], n\rangle\left\langle\nabla_{n} Y, X\right\rangle_{a}-\langle[X, Y], X\rangle\left\langle\nabla_{X} Y, X\right\rangle_{a} \\
-\langle[X, Y], Y\rangle\left\langle\nabla_{Y} Y, X\right\rangle_{a} \\
=\frac{1}{2}\langle[X, Y], n\rangle(-\langle[n, Y], X\rangle+\langle[n, X], Y\rangle+a\langle[Y, X], n\rangle) \\
\quad-\langle[X, Y], X\rangle^{2}-\langle[X, Y], Y\rangle^{2}
\end{gathered}
$$

Summing this up, the sectional curvature of $\xi$ is equal to:

$$
\begin{aligned}
K(\xi)= & -X\langle[Y, X], Y\rangle-\frac{1}{a}\langle[Y, n], Y\rangle\langle[X, n], X\rangle \\
- & \left(Y\langle[X, Y], X\rangle-\frac{1}{4 a}(-a\langle[X, Y], n\rangle+\langle[X, n], Y\rangle+\langle[Y, n], X\rangle)\right. \\
& \cdot(-a\langle[Y, X], n\rangle+\langle[Y, n], X\rangle+\langle[X, n], Y\rangle)) \\
- & \left(-\frac{1}{2}\langle[X, Y], n\rangle(-\langle[n, Y], X\rangle+\langle[n, X], Y\rangle+a\langle[Y, X], n\rangle)\right. \\
& \left.+\langle[X, Y], X\rangle^{2}+\langle[X, Y], Y\rangle^{2}\right)
\end{aligned}
$$

It is straightforward to verify that this gives us the desired expression.

Lemma 3.2 The extrinsic curvature $K_{e}(\xi)$ with respect to $\langle\cdot, \cdot\rangle_{a}$ can be calculated by the following formula:

$$
K_{e}(\xi)=\frac{1}{a}\left(\langle[X, n], X\rangle\langle[Y, n], Y\rangle-\frac{1}{4}(\langle[X, n], Y\rangle+\langle[Y, n], X\rangle)^{2}\right)
$$

Proof Since $X$ and $Y$ are unit vectors, the extrinsic curvature is given by:

$$
K_{e}(\xi)=B(X, X) B(Y, Y)-B(X, Y)^{2}
$$

By the definition of $B$, the extrinsic curvature is equal to:

$$
K_{e}(\xi)=\left\langle\nabla_{X} X, \frac{n}{\sqrt{a}}\right\rangle_{a}\left\langle\nabla_{Y} Y, \frac{n}{\sqrt{a}}\right\rangle_{a}-\frac{1}{4}\left\langle\nabla_{X} Y+\nabla_{Y} X, \frac{n}{\sqrt{a}}\right\rangle_{a}^{2}
$$


Apply the Koszul formula to

$$
\left\langle\nabla_{X} X, \frac{n}{\sqrt{a}}\right\rangle_{a}, \quad\left\langle\nabla_{Y} Y, \frac{n}{\sqrt{a}}\right\rangle_{a} \quad \text { and } \quad\left\langle\nabla_{X} Y+\nabla_{Y} X, \frac{n}{\sqrt{a}}\right\rangle_{a}
$$

to obtain:

$$
\begin{aligned}
K_{e}(\xi)= & \frac{1}{a}\left(\langle[X, n], X\rangle_{a}\langle[Y, n], Y\rangle_{a}-\frac{1}{4}\left(\frac{1}{2}\langle[X, Y], n\rangle_{a}-\frac{1}{2}\langle[X, n], Y\rangle_{a}\right.\right. \\
& \left.\left.-\frac{1}{2}\langle[Y, n], X\rangle_{a}-\frac{1}{2}\langle[X, Y], n\rangle_{a}-\frac{1}{2}\langle[Y, n], X\rangle_{a}-\frac{1}{2}\langle[X, n], Y\rangle_{a}\right)^{2}\right) \\
= & \frac{1}{a}\left(\langle[X, n], X\rangle\langle[Y, n], Y\rangle-\frac{1}{4}(\langle[X, n], Y\rangle+\langle[Y, n], X\rangle)^{2}\right)
\end{aligned}
$$

Summing the extrinsic curvature of $\xi$ with the sectional curvature gives us the Gaussian curvature of the plane distribution $\xi$.

Lemma 3.3 The Gaussian curvature $K_{G}(\xi)$ can be calculated by the formula:

$$
\begin{aligned}
K_{G}(\xi)=K(\xi)+K_{e}(\xi) & \\
=-\frac{3}{4} a\langle[X, Y], n\rangle^{2}+ & \\
& -\langle[\langle[X, Y], Y\rangle-Y\langle[X, Y], X\rangle \\
& \left.-\langle[X, Y], Y\rangle^{2}\right) \\
+ & \frac{1}{2}\langle[X, Y], n\rangle(-\langle[n, Y], X\rangle+\langle[n, X], Y\rangle)
\end{aligned}
$$

Remark 3.4 If $\xi$ is integrable then $\langle[X, Y], n\rangle=0$ and

$$
K_{G}(\xi)=X\langle[X, Y], Y\rangle-Y\langle[X, Y], X\rangle-\langle[X, Y], X\rangle^{2}-\langle[X, Y], Y\rangle^{2}
$$

is nothing else as the expression of the Gaussian curvature of the leaves of $\xi$ written in the local frame tangent to the leaves.

Lemma 3.5 Let $(X, Y, n)$ be a framing on $M$. Assume that distribution spanned by $n$ and $Y$ is a contact structure. Then there is a metric on $M$ such that extrinsic curvature of the distribution spanned by $X$ and $Y$ is strictly less than zero.

Proof Fix a metric $\langle\cdot, \cdot\rangle$ such that the framing is orthonormal. Let $\xi$ be a distribution spanned by vector fields $X$ and $Y$. Stretch the metric along $X$ by a constant factor $\lambda^{2}$ and along $Y$ by a constant factor $1 / \lambda^{2}$. Let's denote this metric by $\langle\cdot, \cdot\rangle_{\lambda}$. Calculate 
the extrinsic curvature of $\xi$ with respect to this metric:

$$
\begin{aligned}
K_{e}(\eta)= & \langle[n, X], X\rangle_{\lambda}\langle[n, Y], Y\rangle_{\lambda}-\frac{1}{4}\left(\langle[n, X], Y\rangle_{\lambda}+\langle[n, Y], X\rangle_{\lambda}\right)^{2} \\
= & \lambda^{2}\langle[n, X], X\rangle \frac{1}{\lambda^{2}}\langle[n, Y], Y\rangle-\frac{1}{4}\left(\frac{1}{\lambda^{2}}\langle[n, X], Y\rangle+\lambda^{2}\langle[n, Y], X\rangle\right)^{2} \\
= & \langle[n, X], X\rangle\langle[n, Y], Y\rangle-\frac{1}{4}\left(\frac{1}{\lambda^{2}}\langle[n, X], Y\rangle+\lambda^{2}\langle[n, Y], X\rangle\right)^{2} \\
= & \langle[n, X], X\rangle\langle[n, Y], Y\rangle-\frac{1}{4 \lambda^{4}}\langle[n, X], Y\rangle^{2}-\frac{1}{2}\langle[n, X], Y\rangle\langle[n, Y], X\rangle \\
& \quad-\frac{\lambda^{4}}{4}\langle[n, Y], X\rangle^{2}
\end{aligned}
$$

Since $M$ is compact there is a positive constant $C$ such that:

$$
\left|\langle[n, X], X\rangle\langle[n, Y], Y\rangle-\frac{1}{2}\langle[n, X], Y\rangle\langle[n, Y], X\rangle\right|<C
$$

We assumed that distribution spanned by vector fields $n$ and $Y$ is a contact structure. The form $\alpha(*)=\langle *, X\rangle$ is a contact form of this distribution, so $\langle[n, Y], X\rangle=$ $\alpha([n, Y]) \neq 0$. Since $M$ is compact there is an $\varepsilon$ such that:

This means that

$$
\begin{array}{r}
\mid\langle[n, Y], X|>\varepsilon \\
K_{e}(\eta)<C-\frac{\lambda^{4} \varepsilon^{2}}{4} .
\end{array}
$$

This expression is strictly negative for some sufficiently large $\lambda$.

Corollary 3.6 Assume that $\xi$ is a transversally orientable contact structure with the Euler class zero on $M$. Then there is a metric on $M$ such that the extrinsic curvature of $\xi$ is a strictly negative function.

Proof Let $n$ be a vector field on $M$ transverse to $\xi$. Since $e(\xi)=0$, the distribution $\xi$ is trivial and has two nowhere zero sections, say $X$ and $Y$.

Choose some positive number $\varepsilon$ and consider a distribution $\eta$ spanned by the vector fields $X$ and $Y+\varepsilon n$. It is obvious that for all $\varepsilon$ the distribution $\eta$ is transverse to $\xi$ and is a contact structure for some sufficiently small $\varepsilon$. Therefore, we can apply Lemma 3.5 to the framing $(X, Y, Y+\varepsilon n)$ to get a desired metric. 


\section{Prescribing the sectional curvature of $\xi$}

Theorem A Let $\xi$ be a transversally orientable contact structure on a closed orientable 3-manifold $M$. For any smooth strictly negative function $f$, there is a metric on $M$ such that $f$ is the sectional curvature of $\xi$.

Proof Since $\xi$ is transversally orientable, there is a globally defined vector field $n$ which is transverse to $\xi$. Fix some Riemannian metric $\langle\cdot, \cdot\rangle$ on $M$ such that $n$ is a unit normal vector field. Consider a finite cover of $M$ by the open sets $U_{\alpha}$ such that for each $\alpha$ there is an open set $U_{\alpha}^{\prime}$ for which $\bar{U}_{\alpha} \subset U_{\alpha}^{\prime}$ and $\left.\xi\right|_{U_{\alpha}^{\prime}}$ is a trivial fibration.

In each $U_{\alpha}^{\prime}$ choose an orthonormal framing $\left(X_{\alpha}, Y_{\alpha},\left.n\right|_{U_{\alpha}^{\prime}}\right)$. Consider the stretching $\langle\cdot, \cdot\rangle_{a}$ of $\langle\cdot, \cdot\rangle$ along $n$ by a positive function $a$.

According to Lemma 3.1 the sectional curvature $K(\xi)$ on $U_{\alpha}^{\prime}$ can be rewritten in the following way:

$$
K(\xi)=-\frac{3}{4} a\left\langle\left[X_{\alpha}, Y_{\alpha}\right], n\right\rangle^{2}+P_{\alpha}+\frac{1}{a} Q_{\alpha}
$$

where $P_{\alpha}$ and $Q_{\alpha}$ are functions on $U_{\alpha}^{\prime}$ independent of $a$.

Since $\xi$ is a contact structure and $U_{\alpha}$ has a compact closure, $\left\langle\left[X_{\alpha}, Y_{\alpha}\right], n\right\rangle^{2}$ is bounded below by some positive constant $\varepsilon$ and the functions $P_{\alpha}$ and $Q_{\alpha}$ are bounded from above. Therefore there is a sufficiently large $D_{\alpha}$ such that the equation

$$
-\frac{3}{4} a\left\langle\left[X_{\alpha}, Y_{\alpha}\right], n\right\rangle^{2}+P_{\alpha}+\frac{1}{a} Q_{\alpha}=f D_{\alpha}
$$

has a strictly positive solution $a_{\alpha}\left(D_{\alpha}\right)$. Notice, that for any $D>D_{\alpha}$ this equation still has a positive solution $a_{\alpha}(D)$. Let $D_{0}=\max _{\alpha}\left\{D_{\alpha}\right\}$. Solve the equation above for $D_{0}$ in each chart $U_{\alpha}$. Let $a_{\alpha}=a_{\alpha}\left(D_{0}\right)$.

We claim that $a_{\alpha}$ constructed this way does not depend on the choice of the orthonormal framing $\left(X_{\alpha}, Y_{\alpha},\left.n\right|_{U_{\alpha}}\right)$. Let $\left(X_{\alpha}^{\prime}, Y_{\alpha}^{\prime},\left.n\right|_{U_{\alpha}}\right)$ be any other orthonormal framing on $\left.\xi\right|_{U_{\alpha}}$. This defines a map

$$
\phi_{\alpha}: U_{\alpha} \rightarrow O(2)
$$

which maps a point $p \in U_{\alpha}$ to the transition matrix $\phi_{\alpha}(p)$ between two framings $\left(X_{\alpha}^{\prime}, Y_{\alpha}^{\prime}\right)$ and $\left(X_{\alpha}, Y_{\alpha}\right)$ on $\xi$. We have

$$
\begin{aligned}
\left\langle\left[X_{\alpha}^{\prime}, Y_{\alpha}^{\prime}\right], n\right\rangle^{2} & =\left(d \eta\left(X_{\alpha}^{\prime}, Y_{\alpha}^{\prime}\right)\right)^{2}=\left(d \eta\left(\phi_{\alpha} X_{\alpha}, \phi_{\alpha} Y_{\alpha}\right)\right)^{2}=\operatorname{det} \phi_{\alpha}^{2}\left(d \eta\left(X_{\alpha}, Y_{\alpha}\right)\right)^{2} \\
& =\operatorname{det} \phi_{\alpha}^{2}\left\langle\left[X_{\alpha}, Y_{\alpha}\right], n\right\rangle^{2}=\left\langle\left[X_{\alpha}, Y_{\alpha}\right], n\right\rangle^{2}
\end{aligned}
$$

where $\eta$ is a $1-$ form defined by $\eta(*)=\langle *, n\rangle$. Therefore, $\left\langle\left[X_{\alpha}, Y_{\alpha}\right], n\right\rangle^{2}$ is independent of the choice of orthonormal framing. The expression $(1 / a) Q_{\alpha}=-K_{e}(\xi)$ also does 
not depend on the choice of the trivialization. Finally the sectional curvature $K(\xi)$ is independent of the framing. It is obvious that the right hand side of

$$
P_{\alpha}=K(\xi)-\frac{1}{a} Q_{\alpha}+\frac{3}{4} a\left\langle\left[X_{\alpha}, Y_{\alpha}\right], n\right\rangle^{2}
$$

does not depend on the choice of framing, so does $P_{\alpha}$.

Therefore, the functions $a_{\alpha}$ agree on the overlaps and define a global function $a$ on $M$. The sectional curvature of $\xi$ in the metric $\langle\cdot, \cdot\rangle_{a}$ is $f D_{0}$. Consider the metric $\langle\cdot, \cdot\rangle_{0}=\left(1 / D_{0}\right)\langle\cdot, \cdot\rangle_{a}$. It is easy to calculate, that the sectional curvature of $\xi$ in this metric is equal to $f$.

Corollary 4.1 For any transversally orientable contact structure on a closed orientable 3 -manifold, there is a metric on $M$,such that the sectional curvature of $\xi$ in this metric is equal to -1 .

Theorem B Let $\xi$ be a transversally orientable contact structure on $M$ with Euler class zero. Then for any smooth function $f$, there is a metric on $M$ such that $f$ is a sectional curvature of $\xi$.

Proof Since the Euler class of $\xi$ is zero, there is a contact structure $\eta$, which is transverse to $\xi$. According to the Corollary 3.6, there is a metric $\langle\cdot, \cdot\rangle$ in which the extrinsic curvature of $\xi$ is a strictly negative function. Let $n$ be a unit normal vector field with respect to this metric.

Consider the stretching of $\langle\cdot, \cdot\rangle$ along $n$ by a positive function $a$. According to Lemma 3.1 , we have to find $a$ to satisfy the equation

$$
-\frac{3}{4} a\langle[X, Y], n\rangle^{2}+P-\frac{1}{4 a} K_{e}(\xi)=f
$$

where $P$ is a function on $M$ which is independent of $a$.

But since $-K_{e}(\xi)>0$ this equation always has a strictly positive solution $a$. This completes the proof of the theorem.

Remark 4.2 In the proof of Theorem B it is crucial that $\xi$ is a contact structure. At points where $\langle[X, Y], n\rangle=0$ the equation may not have any positive solutions.

Example 4.3 (Propeller construction [6]) Consider the following pair of contact structures on $\mathbb{T}^{3}$ :

$$
\begin{aligned}
& \xi=\operatorname{Ker}(\alpha=\cos z d x-\sin z d y+d z) \\
& \eta=\operatorname{Ker}(\beta=\cos z d x+\sin z d y)
\end{aligned}
$$


It is easy to verify, that $\xi$ is transverse to $\eta$ and we get a bi-contact structure. From Theorem $\mathrm{B}$, there is a metric on $\mathbb{T}^{3}$ such that $\xi$ has a positive sectional curvature. This is an example of a tight contact structure of positive sectional curvature.

Example 4.4 (Overtwisted contact structures of positive sectional curvature) Let $\xi$ be any contact structure with the Euler class zero on $M$. It is known (see Geiges [3]) that if we apply a full Lutz twist to this contact structure, the resulting contact structure is overtwisted and has Euler class zero. From Theorem B, it has a positive sectional curvature for some choice of metric on $M$.

\section{Uniformization of contact structures on 3-manifolds}

The same technique as in Theorem A can be applied to the Gaussian curvature of contact structures on three-manifolds.

Theorem C Let $\xi$ be a transversally orientable contact structure on a closed orientable 3-manifold $M$. For any smooth strictly negative function $f$, there is a metric on $M$ such that $f$ is the Gaussian curvature of $\xi$.

Proof Same as Theorem A. The only difference is that in the present case the equation which needs to be solved in each trivializing chart is:

$$
K_{G}(\xi)=-\frac{3}{4} a\left\langle\left[X_{\alpha}, Y_{\alpha}\right], n\right\rangle^{2}+P_{\alpha}=f D_{0}
$$

Corollary 5.1 (Uniformization of contact structures) For every transversally orientable contact structure $\xi$ on $M$, there is a metric such that $K_{G}(\xi)=-1$.

Example 5.2 (Contact structure with $K_{G}(\xi)=1$ ) Consider the unit sphere $S^{3} \subset \mathbb{C}^{2}$ with a bi-invariant metric. The standard contact structure on $S^{3}$ is defined as the kernel of the 1 -form

$$
\alpha=\sum_{i=1}^{2}\left(x_{i} d y_{i}-y_{i} d x_{i}\right),
$$

restricted from $\mathbb{C}^{2}$ to $S^{3}$. This contact structure is orthogonal to a left-invariant vector field and therefore is left-invariant. Let $(X, Y)$ be a pair of orthonormal left-invariant sections of $\xi$. Since the metric is bi-invariant,

$$
\nabla_{S} T=\frac{1}{2}[S, T]
$$

for any left-invariant vector fields on $S^{3}$. Therefore the second fundamental form of $\xi$ vanishes and $K_{G}(\xi)=K(\xi)=1$. 


\section{References}

[1] A Candel, Uniformization of surface laminations, Ann. Sci. École Norm. Sup. (4) 26 (1993) 489-516 MR1235439

[2] S S Chern, R S Hamilton, On Riemannian metrics adapted to three-dimensional contact manifolds, from: "Workshop (Bonn, 1984)", Lecture Notes in Math. 1111, Springer, Berlin (1985) 279-308 MR797427 With an appendix by A Weinstein

[3] H Geiges, Contact geometry, from: "Handbook of differential geometry. Vol. II", Elsevier/North-Holland, Amsterdam (2006) 315-382 MR2194671

[4] J M Lee, T H Parker, The Yamabe problem, Bull. Amer. Math. Soc. (N.S.) 17 (1987) 37-91 MR888880

[5] J Lohkamp, Metrics of negative Ricci curvature, Ann. of Math. (2) 140 (1994) 655683 MR1307899

[6] Y Mitsumatsu, Anosov flows and non-Stein symplectic manifolds, Ann. Inst. Fourier (Grenoble) 45 (1995) 1407-1421 MR1370752

[7] B L Reinhart, The second fundamental form of a plane field, J. Differential Geom. 12 (1977) 619-627 (1978) MR512930

Department of Geometry, Institute for Low Temperature Physics and Engineering 47 Lenin Ave, Kharkov 61103, Ukraine

vkrouglov@gmail.com

Received: 4 February 2008 Revised: 24 July 2008 